\title{
Algebraic structures of $F$-manifolds via pre-Lie algebras
}

\section{Vladimir Dotsenko ${ }^{1}$}

Received: 5 June 2018 / Accepted: 12 August 2018 / Published online: 22 August 2018

(c) Fondazione Annali di Matematica Pura ed Applicata and Springer-Verlag GmbH Germany, part of Springer Nature 2018

\begin{abstract}
We relate the operad FMan controlling the algebraic structure on the tangent sheaf of an $F$-manifold (weak Frobenius manifold) defined by Hertling and Manin to the operad PreLie of pre-Lie algebras: for the filtration of PreLie by powers of the ideal generated by the Lie bracket, the associated graded object is FMan.
\end{abstract}

Keywords $F$-manifold $\cdot$ Pre-Lie algebra $\cdot$ Operad $\cdot$ Minimal model

Mathematics Subject Classification 18D50 - 18G55 · 53D45 · 68Q42

\section{Introduction}

The notion of an $F$-manifold (weak Frobenius manifold) was introduced by Hertling and Manin [8] as a relaxation of the notion of a Frobenius manifold. By definition, an $F$-manifold is a pair $(M,-\circ-)$ consisting of a smooth supermanifold $M$ and a smooth bilinear commutative associative product - - - on the tangent sheaf $T M$ satisfying the condition

$$
P_{X_{1} \circ X_{2}}\left(X_{3}, X_{4}\right)=X_{1} \circ P_{X_{2}}\left(X_{3}, X_{4}\right)+(-1)^{\left|X_{1}\right|\left|X_{2}\right|} X_{2} \circ P_{X_{1}}\left(X_{3}, X_{4}\right),
$$

where $P_{X_{1}}\left(X_{2}, X_{3}\right)=\left[X_{1}, X_{2} \circ X_{3}\right]-\left[X_{1}, X_{2}\right] \circ X_{3}-(-1)^{\left|X_{1}\right|\left|X_{2}\right|} X_{2} \circ\left[X_{1}, X_{3}\right]$ measures to what extent the product $-\circ-$ and the usual Lie bracket of vector fields fail the Poisson algebra axioms. Any Frobenius manifold is an $F$-manifold; any $F$-manifold for which the product - o - is semisimple can be made into a Frobenius manifold [8]. A very deep operadic result on $F$ manifolds was established by Merkulov [19]: any homotopy Gerstenhaber algebra gives rise to an $F$-manifold (in fact to a much richer structure including higher operations). However, a very fundamental problem of a reasonable description of the operad FMan encoding " $F$ manifold algebras", that is algebras with an associative commutative product and a Lie bracket satisfying the Hertling-Manin condition, has never been addressed. On the one hand, it is not surprising, since the Hertling-Manin condition is a cubic relation, and as such, the operad FMan is completely outside the scope of applicability of traditional methods of the operad theory like the Koszul duality theory, so there are no readily available methods to study it,

$\bowtie$ Vladimir Dotsenko

vdots@maths.tcd.ie

1 School of Mathematics, Trinity College, Dublin 2, Ireland 
as remarked by Manin in [14, Sect. 3.3, Remark] where the question of studying the operad FMan is emphasised. On the other hand, this algebraic structure appears as fundamental as the one controlled by the Poisson operad, and its satisfactory algebraic description is highly desirable.

In this paper, we give a very direct description of the operad FMan. Quite surprisingly, this operad turns out to be intimately related to one of the most famous operads appearing in the literature, the operad PreLie of pre-Lie algebras [4] that controls bilinear products - - on a graded vector space $V$ satisfying the condition

$$
\left(X_{1} \cdot X_{2}\right) \cdot X_{3}-X_{1} \cdot\left(X_{2} \cdot X_{3}\right)=(-1)^{\left|X_{2}\right|\left|X_{3}\right|}\left(\left(X_{1} \cdot X_{3}\right) \cdot X_{2}-X_{1} \cdot\left(X_{3} \cdot X_{2}\right)\right) \text {. }
$$

This condition implies that the bracket $\left[X_{1}, X_{2}\right]=X_{1} \cdot X_{2}-(-1)^{\left|X_{1}\right|\left|X_{2}\right|} X_{2} \cdot X_{1}$ satisfies the Jacobi identity. The slogan promoted by this note is " $F$-manifold algebras are the same to pre-Lie algebras as Poisson algebras to associative algebras." (The word "algebras" is used in that slogan four times to emphasise that we consider algebraic properties of the structure on the tangent sheaf of an $F$-manifold and ignore the geometry of the arguments, the vector fields.)

In fact, there exists a commutative diagram of operads

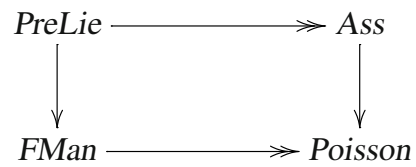

where the vertical arrows are isomorphisms on the level of $\mathbb{S}$-modules (but not on the level of operads). Note that in contrast to the operad FMan the defining relations of the operad PreLie are quadratic, and the symmetrised pre-Lie product $X_{1} \circ X_{2}=X_{1} \cdot X_{2}+(-1)^{\left|X_{1}\right|\left|X_{2}\right|} X_{2} \cdot X_{1}$ does not satisfy any identities at all, as established in [2]. Our approach substantially relies on shuffle operads and rewriting methods for operads. We introduce and use two new general notions: an almost composite product and an almost distributive law.

This is a short note, and we do not intend to overload it with excessive recollections. For relevant information on symmetric operads and Koszul duality, we refer the reader to the monograph [12] and, for information on shuffle operads, Gröbner bases and rewriting systems to the monograph [3].

All operads in this paper are defined over a field $\mathbb{k}$ of characteristic zero. We assume all operads reduced $(\mathcal{P}(0)=0)$ and connected $(\mathcal{P}(1)=\mathbb{k})$. The (co)augmentation (co)ideal of an (co)operad $\mathcal{P}$ is denoted $\overline{\mathcal{P}}$. The Koszul dual of a quadratic operad $\mathcal{P}$ is denoted $\mathcal{P}$ !. When writing down elements of operads, we use arguments $a_{1}, \ldots, a_{n}$ as placeholders and capital Latin letters $X_{1}, \ldots, X_{n}$ as arguments (belonging to actual graded vector spaces on which operads act). Thus, the pre-Lie identity in the operadic form is

$$
\left(a_{1} \cdot a_{2}\right) \cdot a_{3}-a_{1} \cdot\left(a_{2} \cdot a_{3}\right)=\left(a_{1} \cdot a_{3}\right) \cdot a_{2}-a_{1} \cdot\left(a_{3} \cdot a_{2}\right),
$$

and the signs in (1) arise from applying this to a decomposable tensor $X_{1} \otimes X_{2} \otimes X_{3}$ and using the standard Koszul sign rule.

\section{The Lie filtration and $\boldsymbol{F}$-manifold algebras}

We begin with making the statements from Introduction completely clear. Let us begin with a precise operadic definition of the protagonist of this paper. 
Definition 1 The operad FMan of $F$-manifold algebras is generated by a symmetric binary operation - - - and a skew-symmetric binary operation $[-,-]$ satisfying the associativity relation and the Jacobi identity

$$
\begin{aligned}
& \left(a_{1} \circ a_{2}\right) \circ a_{3}=a_{1} \circ\left(a_{2} \circ a_{3}\right), \\
& {\left[\left[a_{1}, a_{2}\right], a_{3}\right]+\left[\left[a_{2}, a_{3}\right], a_{1}\right]+\left[\left[a_{3}, a_{1}\right], a_{2}\right]=0,}
\end{aligned}
$$

and related to each other by the Hertling-Manin relation

$$
\begin{aligned}
{\left[a_{1} \circ a_{2}, a_{3} \circ a_{4}\right]=} & {\left[a_{1} \circ a_{2}, a_{3}\right] \circ a_{4}+\left[a_{1} \circ a_{2}, a_{4}\right] \circ a_{3}+a_{1} \circ\left[a_{2}, a_{3} \circ a_{4}\right] } \\
& +a_{2} \circ\left[a_{1}, a_{3} \circ a_{4}\right]-\left(a_{1} \circ a_{3}\right) \circ\left[a_{2}, a_{4}\right]-\left(a_{2} \circ a_{3}\right) \circ\left[a_{1}, a_{4}\right] \\
& -\left(a_{2} \circ a_{4}\right) \circ\left[a_{1}, a_{3}\right]-\left(a_{1} \circ a_{4}\right) \circ\left[a_{2}, a_{3}\right] .
\end{aligned}
$$

Our next step is to define the filtration of the pre-Lie operad which we shall need.

Definition 2 The Lie filtration $F^{\bullet}$ PreLie of the operad of pre-Lie algebras is defined as the filtration by powers of the ideal generated by the Lie bracket $[-,-]$. In other words, $F^{k}$ PreLie is the span of tree tensors in which at least $k$ vertices are labelled with $[-,-]$.

Now everything is prepared for our main result to be stated.

Theorem 1 The operad FMan is the associated graded object for the Lie filtration: we have an operad isomorphism

$$
\mathrm{gr}_{F} \text { PreLie } \cong \text { FMan }
$$

In particular,

$$
\operatorname{dim} \operatorname{FMan}(n)=n^{n-1},
$$

and the $S_{n}$-module FMan( $(n)$ is isomorphic to the module of rooted trees on $\{1, \ldots, n\}$.

Note that the same filtration can be defined for the operad Ass of associative algebras. In that case, it is well known $[11,17]$ that $\operatorname{gr}_{F}$ Ass $\cong$ Poisson. To prove it, one may first note that a direct computation demonstrates that relations of Poisson hold in $\operatorname{gr}_{F}$ Ass, which leads to a surjective map between these operads. Establishing that this map is an isomorphism can then be done by proving that this operads are of the same size, which requires some extra work. It turns out that a similar strategy, albeit more involved in the "extra work" part, is available in our case. We shall make the first step and establish a lower bound on the operad FMan (a posteriori, this bound will turn out to be sharp) and then develop a general formalism of almost composite products and almost distributive laws needed to complete the proof (note that in the case of the Poisson operad, we are dealing with actual composite products and distributive laws, so the parallelism of proofs is very apparent).

Lemma 1 We have a surjection of operads

$$
\text { FMan } \rightarrow \mathrm{gr}_{F} \text { PreLie. }
$$

Proof Recall that the symmetrised pre-Lie product - o- and the bracket $[-,-]$ in the operad PreLie satisfy the following relation:

$$
\begin{aligned}
& \left(a_{1} \circ a_{2}\right) \circ a_{3}-a_{1} \circ\left(a_{2} \circ a_{3}\right)-a_{1} \circ\left[a_{2}, a_{3}\right]-\left[a_{1}, a_{2}\right] \circ a_{3}-2\left[a_{1}, a_{3}\right] \circ a_{2} \\
& \quad+\left[a_{1}, a_{2} \circ a_{3}\right]+\left[a_{1} \circ a_{2}, a_{3}\right]+\left[\left[a_{1}, a_{3}\right], a_{2}\right]=0,
\end{aligned}
$$


(see [3, Example 5.6.4.1]). Considering it as a relation in a shuffle operad and computing its S-polynomial with itself, one arrives at a relation

$$
\begin{aligned}
- & {\left[a_{1} \circ a_{2}, a_{3}\right] \circ a_{4}-\left[a_{1} \circ a_{2}, a_{4}\right] \circ a_{3}+\left[a_{1}, a_{4}\right] \circ\left(a_{2} \circ a_{3}\right)+\left[a_{1}, a_{3}\right] \circ\left(a_{2} \circ a_{4}\right) } \\
& -\left[a_{1}, a_{3} \circ a_{4}\right] \circ a_{2}+\left[a_{1} \circ a_{2}, a_{3} \circ a_{4}\right]+a_{1} \circ\left(\left[a_{2}, a_{3}\right] \circ a_{4}\right)+a_{1} \circ\left(\left[a_{2}, a_{4}\right] \circ a_{3}\right) \\
& -a_{1} \circ\left[a_{2}, a_{3} \circ a_{4}\right]+\left[\left[a_{1}, a_{3}\right], a_{2}\right] \circ a_{4}+\left[\left[a_{1}, a_{4}\right], a_{2}\right] \circ a_{3}+2\left[\left[a_{1}, a_{4}\right], a_{3}\right] \circ a_{2} \\
& +\left[a_{1},\left[a_{2}, a_{3}\right]\right] \circ a_{4}+\left[a_{1},\left[a_{2}, a_{4}\right]\right] \circ a_{3}+\left[a_{1}, a_{4}\right] \circ\left[a_{2}, a_{3}\right]+\left[a_{1}, a_{3}\right] \circ\left[a_{2}, a_{4}\right] \\
& +\left[a_{1},\left[a_{3}, a_{4}\right]\right] \circ a_{2}-\left[\left[a_{1}, a_{4}\right], a_{2} \circ a_{3}\right]-\left[\left[a_{1}, a_{3}\right], a_{2} \circ a_{4}\right]-\left[a_{1},\left[a_{2}, a_{3}\right] \circ a_{4}\right] \\
& -\left[a_{1},\left[a_{2} a_{4}\right] \circ a_{3}\right]-2\left[\left[\left[a_{1}, a_{4}\right], a_{3}\right], a_{2}\right]-\left[\left[a_{1}, a_{4}\right],\left[a_{2}, a_{3}\right]\right]-\left[\left[a_{1}, a_{3}\right],\left[a_{2}, a_{4}\right]\right] \\
& -\left[\left[a_{1},\left[a_{3}, a_{4}\right]\right], a_{2}\right]=0 .
\end{aligned}
$$

Let us consider the images of both of these relations in $\operatorname{gr}_{F}$ PreLie. The first one is a combination of terms where the bracket is used 0,1 , and 2 times. In the associated graded object, this relation becomes the associativity of the product $-\circ-$. The second one is a combination of terms where the bracket is used 1, 2, and 3 times. In the associated graded object, this relation becomes

$$
\begin{aligned}
& -\left[a_{1} \circ a_{2}, a_{3}\right] \circ a_{4}-\left[a_{1} \circ a_{2}, a_{4}\right] \circ a_{3}+\left[a_{1}, a_{4}\right] \circ\left(a_{2} \circ a_{3}\right) \\
& \quad+\left[a_{1}, a_{3}\right] \circ\left(a_{2} \circ a_{4}\right)-\left[a_{1}, a_{3} \circ a_{4}\right] \circ a_{2} \\
& \quad+\left[a_{1} \circ a_{2}, a_{3} \circ a_{4}\right]+a_{1} \circ\left(\left[a_{2}, a_{3}\right] \circ a_{4}\right)+a_{1} \circ\left(\left[a_{2}, a_{4}\right] \circ a_{3}\right) \\
& -a_{1} \circ\left[a_{2}, a_{3} \circ a_{4}\right]=0,
\end{aligned}
$$

which, modulo associativity, is equivalent to the Hertling-Manin condition. It remains to notice that by a standard polarisation argument [17], the operations - -- and $[-,-]$ generate the operad PreLie, so the operad $\mathrm{gr}_{F}$ PreLie is generated by their cosets, and hence, it is a homomorphic image of the operad FMan.

\section{Almost composite products and almost distributive laws}

In the case of the operad of Poisson algebras, the relation between $-\circ-$ and $[-,-]$ is a rewriting rule allowing to get rid of all occurrences of products inside brackets, showing that there is a surjective map onto the Poisson operad from the composite product Com $\circ$ Lie. In the case of $F$-manifold algebras, one can mimic this approach. For that, we introduce and study a new general operadic construction.

Definition 3 Suppose that $\mathcal{P}=\mathcal{T}(\mathcal{X}) /(\mathcal{R})$ and $\mathcal{Q}=\mathcal{T}(\mathcal{Y}) /(\mathcal{S})$ are two operads. The almost composite product of $\mathcal{P}$ and $\mathcal{Q}$, denoted $\mathcal{P} \nabla_{0} \mathcal{Q}$, is defined as

$$
\mathcal{P} \nabla_{0} \mathcal{Q}:=\mathcal{P} \bigvee \mathcal{Q} /\left(\alpha\left(\beta_{1}, \ldots, \beta_{k}\right): \alpha \in \mathcal{Y}, \beta_{i} \in \mathcal{X}\right)
$$

Remark 1 The usual composite product $\mathcal{P} \circ \mathcal{Q}$ of the underlying $\mathbb{S}$-modules of $\mathcal{P}$ and $\mathcal{Q}$ is the underlying $\mathbb{S}$-module of the operad

$$
\mathcal{P} \vee_{0} \mathcal{Q}:=\mathcal{P} \bigvee \mathcal{Q} /(\alpha(\beta, \text { id }, \ldots, \text { id }): \alpha \in \mathcal{Y}, \beta \in \mathcal{X}),
$$

whose relations are known as the trivial distributive law between $\mathcal{P}$ and $\mathcal{Q}$ (see [12, Sect. 8.6.4]) which explains our terminology. Note that unlike the trivial distributive laws, almost composite products are not defined by quadratic relations, unless $\mathcal{Q}$ is generated by 
unary operations. This takes these operads outside the scope of commonly used methods of operad theory.

We shall now establish two general results about almost composite products, which are analogous to corresponding results about trivial distributive laws. First, we shall show that the almost composite product of two operads $\mathcal{P}$ and $\mathcal{Q}$ is an upper bound on a certain class of quotients of $\mathcal{P} \vee \mathcal{Q}$, in the same way as the trivial distributive law is an upper bound for general rewriting rules used to define distributive laws between operads $[12,16]$.

Proposition 1 Let $\mathcal{P}=\mathcal{T}(\mathcal{X}) /(\mathcal{R})$ and $\mathcal{Q}=\mathcal{T}(\mathcal{Y}) /(\mathcal{S})$ be two operads, and let $\mathcal{O}$ be an operad of the form

$$
\mathcal{O}:=\mathcal{P} \bigvee \mathcal{Q} /\left(\alpha\left(\beta_{1}, \ldots, \beta_{k}\right)-f\left(\alpha\left(\beta_{1}, \ldots, \beta_{k}\right)\right): \alpha \in \mathcal{Y}, \beta_{i} \in \mathcal{X}\right),
$$

where $f: \mathcal{Y} \circ \mathcal{X} \rightarrow \mathcal{P} \bigvee \mathcal{Q}$ is a linear map whose image is contained in the right ideal generated by $\mathcal{X}$. Then for each $n \geq 1$, there is a surjection of vector spaces

$$
\left(\mathcal{P} \nabla_{0} \mathcal{Q}\right)(n) \rightarrow \mathcal{O}(n) .
$$

Proof Let us view the shuffle operads associated with $\mathcal{P} \nabla_{0} \mathcal{Q}$ and $\mathcal{O}$ as quotients of the free shuffle operad generated by $\mathcal{X} \oplus \mathcal{Y}$. We fix some admissible order $\prec_{0}$ of shuffle tree monomials.

We first note that from the algorithm of computing the reduced Gröbner basis for a given operad, it is clear that the reduced Gröbner basis for the operad $\mathcal{P} \nabla_{0} \mathcal{Q}$ consists of linear combinations of monomials where vertices labelled by elements of $\mathcal{Y}$ are "closer to the leaves", that is, there is no vertex labelled by an element from $\mathcal{Y}$ which has a child labelled by an element of $\mathcal{X}$.

Let us now examine the operad $\mathcal{O}$. The situation with this operad is more complicated: generally, there is no choice of an admissible order for which $\alpha\left(\beta_{1}, \ldots, \beta_{k}\right)$ is the leading term of $\alpha\left(\beta_{1}, \ldots, \beta_{k}\right)-f\left(\alpha\left(\beta_{1}, \ldots, \beta_{k}\right)\right)$. For that reason, we need to invoke a rewriting system argument. Let us define the following order on shuffle tree monomials: $T \prec T^{\prime}$ if

- $n(T)>n\left(T^{\prime}\right)$, where $n(T)$ is the number of pairs of internal vertices $\left(v, v^{\prime}\right)$ of $T$, where $v$ is on the way from the root of $T$ to $v^{\prime}$, the label of $v$ is from $\mathcal{X}$, and the label of $v^{\prime}$ is from $\mathcal{Y}$;

- $n(T)=n\left(T^{\prime}\right)$, and $T \prec_{0} T^{\prime}$.

This order is not a monomial order. However, it is a total order, so it certainly allows to convert relations into rewriting rules (so every set of relations gives rise to a rewriting system), and it is a well order (so every rewriting system is terminating). Note that for the operad $\mathcal{P} \nabla_{0} \mathcal{Q}$ only tree monomials $T$ with $n(T)=0$ appear in the process of Knuth-Bendix completion of the corresponding rewriting system [9], so the direction of all relations is dictated by $\prec_{0}$, and the result of the completion is the reduced Gröbner basis.

Let us now apply the Knuth-Bendix completion procedure to relations of the operad $\mathcal{O}$. It is clear that when we consider the critical pairs coming from the mixed $\mathcal{P}-\mathcal{Q}$-relations and the $\mathcal{Q}$-relations, the computation mimics the one we performed for the operad $\mathcal{P} \nabla_{0} \mathcal{Q}$, adding some extra terms for which the parameter $n(T)$ is higher; thus, these terms are smaller with respect to the order $\prec$. This means that whenever the Knuth-Bendix procedure produced a new rewriting rule for $\mathcal{P} \nabla_{0} \mathcal{Q}$, it produces a new rewriting rule for $\mathcal{O}$, and the left-hand side of that rule is the same. (In principle, completely new rewriting rules may arise here: if a critical pair does not produce a new rewriting rule for $\mathcal{P} \nabla_{0} \mathcal{Q}$, it still may produce one for $\mathcal{O}$.) By contrast, the critical pairs coming from the mixed $\mathcal{P}-\mathcal{Q}$-relations and the $\mathcal{P}$-relations 
contribute nothing in the case of the operad $\mathcal{P} \nabla_{0} \mathcal{Q}$, but may result in new Gröbner basis elements for $\mathcal{O}$.

We observe that the left-hand sides of the rewriting rule set of normal forms for the component $\mathcal{O}$ include all the left- hand sides of the rewriting rule set of normal forms for the component $\mathcal{P} \vee \mathcal{Q}$, so the set of normal forms for the operad $\mathcal{O}$ is a subset of the set of the normal forms for $\left(\mathcal{P} \nabla_{0} \mathcal{Q}\right)(n)$, which proves our claim.

The analogy with distributive laws mentioned above suggests the following definition.

Definition 4 Let $\mathcal{P}=\mathcal{T}(\mathcal{X}) /(\mathcal{R})$ and $\mathcal{Q}=\mathcal{T}(\mathcal{Y}) /(\mathcal{S})$ be two operads, and let $\mathcal{O}$ be an operad of the form

$$
\mathcal{O}:=\mathcal{P} \bigvee \mathcal{Q} /\left(\alpha\left(\beta_{1}, \ldots, \beta_{k}\right)-f\left(\alpha\left(\beta_{1}, \ldots, \beta_{k}\right)\right): \alpha \in \mathcal{Y}, \beta_{i} \in \mathcal{X}\right),
$$

where $f: \mathcal{Y} \circ \mathcal{X} \rightarrow \mathcal{P} \vee \mathcal{Q}$ is a linear map whose image is contained in the right ideal generated by $\mathcal{X}$. The map $f$ is said to be an almost distributive law between $\mathcal{P}$ and $\mathcal{Q}$ if for each $n$ we have an isomorphism of $\mathbb{S}$-modules

$$
\left(\mathcal{P} \nabla_{0} \mathcal{Q}\right)(n) \cong \mathcal{O}(n) .
$$

Let us now determine the minimal model of the almost composite product of two Koszul operads. This result is inspired by both the computation of the minimal model of the trivial distributive law between two Koszul operads [12, Prop. 8.6.3] and the approach to infinity morphisms of strong homotopy algebras using "homotopy Koszul operads" [20].

Proposition 2 Let $\mathcal{P}=\mathcal{T}(\mathcal{X}) /(\mathcal{R})$ and $\mathcal{Q}=\mathcal{T}(\mathcal{Y}) /(\mathcal{S})$ be two Koszul operads. Consider the endomorphism d of the free operad $\mathcal{T}\left(s^{-1} \overline{\mathcal{Q} !} \oplus \overline{\mathcal{Q} !} \oplus s^{-1} \overline{\mathcal{P} !}\right)$ defined on the generators as follows:

- on the first group of generators, $d\left(s^{-1} \lambda\right)=d_{\Omega(\mathcal{Q} !)}\left(s^{-1} \lambda\right)$,

- on the second group of generators, $d(\lambda)=s^{-1} \lambda-(\mathrm{id} \otimes s) d_{\Omega\left(\mathcal{Q}^{!}\right)}\left(s^{-1} \lambda\right)$,

- on the third group of generators, $d\left(s^{-1} \mu\right)=d_{\Omega(\mathcal{P} !)}\left(s^{-1} \mu\right)$.

Then $d^{2}=0$. Moreover, the suboperad of $\mathcal{T}\left(s^{-1} \overline{\mathcal{Q}^{!}} \oplus \overline{\mathcal{Q} !} \oplus s^{-1} \overline{\mathcal{P}^{!}}\right)$generated by

$$
s^{-1} \overline{\mathcal{Q}^{!}} \oplus s^{-1} \overline{\mathcal{P} !} \oplus\left(\overline{\mathcal{Q}^{!}} \circ\left(s^{-1} \overline{\mathcal{P}^{!}}\right)\right)
$$

is a free operad and a d-invariant subspace, and the quasi-free operad

$$
\left(\mathcal{T}\left(s^{-1} \overline{\mathcal{Q}^{!}} \oplus s^{-1} \overline{\mathcal{P}^{!}} \oplus\left(\overline{\mathcal{Q}^{!}} \circ\left(s^{-1} \overline{\mathcal{P}^{!}}\right)\right)\right), d\right)
$$

is a minimal model of the operad $\mathcal{P} \nabla_{0} \mathcal{Q}$.

Proof A direct inspection confirms that $d^{2}=0$ (note that this is only needed to check on the second group of generators, as on the two other groups it follows from the fact that the cobar complex of a cooperad is a chain complex). It is also immediate to check that the suboperad $s^{-1} \overline{\mathcal{Q}^{!}} \oplus s^{-1} \overline{\mathcal{P}^{!}} \oplus\left(\overline{\mathcal{Q}^{!}} \circ\left(s^{-1} \overline{\mathcal{P} !}\right)\right)$ is $d$-invariant; once again, it is clear that for the generators of the first and the second group, their images under $d$ are made of elements of the same kind, so only the generators of $\overline{\mathcal{Q} !} \circ\left(s^{-1} \overline{\mathcal{P} !}\right)$ need to be inspected. Also, this suboperad is free, since every element can be uniquely represented as a composite of generators; for that, it is useful to note that $\overline{\mathcal{Q}^{!}}$(without $s^{-1}$ ) only appears in the third group of generators. 
Let us compute the homology of the operad

$$
\left(\mathcal{T}\left(s^{-1} \overline{\mathcal{Q}^{!}} \oplus s^{-1} \overline{\mathcal{P} !} \oplus\left(\overline{\mathcal{Q}^{!}} \circ\left(s^{-1} \overline{\mathcal{P}^{!}}\right)\right)\right), d\right) .
$$

For that, we introduce a weight grading on this operad defined by assigning weight 0 to generators from $s^{-1} \overline{\mathcal{P} !}$ and weight 1 to generators from both $s^{-1} \overline{\mathcal{Q} !}$ and $\overline{\mathcal{Q} !} \circ\left(s^{-1} \overline{\mathcal{P} !}\right)$. Note that the contributions of $d_{\Omega(\mathcal{P} !)}$ to $d$ (those appear in the images under $d$ of generators from $s^{-1} \overline{\mathcal{P} !}$ and $\overline{\mathcal{Q} !} \circ\left(s^{-1} \overline{\mathcal{P} !}\right)$ do not change weight, and all other contributions to $d$ increase weight by at least one. Thus, we are dealing with a filtered chain complex, and we may consider the corresponding spectral sequence. The differential of the first page of that spectral sequence kills all the higher homotopies for $\mathcal{P}$; thus, homology of that differential is identified with the operad

$$
\mathcal{T}\left(\mathcal{X} \oplus s^{-1} \overline{\mathcal{Q} !} \oplus(\overline{\mathcal{Q} !} \circ \mathcal{X})\right) /(\mathcal{R}),
$$

with the obvious differential derived from $d$. To compute the homology of that differential, we introduce another filtration defined by assigning weight 0 to generators from $\mathcal{X}$ and weight 1 to generators from both $s^{-1} \overline{\mathcal{Q}^{!}}$and $\overline{\mathcal{Q}^{!}} \circ \mathcal{X}$. Note that for each generator, the contribution of the map $\lambda \mapsto s^{-1} \lambda$ does not change weight, and the contributions of $d_{\Omega(\mathcal{Q} !)}$ increase the weight by at least one. The differential of the first page of the spectral sequence of this new filtered complex has the homology

$$
\mathcal{T}\left(\mathcal{X} \oplus s^{-1} \overline{\mathcal{Q}^{!}}\right) /\left(\mathcal{R} \oplus\left(s^{-1} \overline{\mathcal{Q}^{!}} \circ \mathcal{X}\right)\right),
$$

with the differential $\left(0, d_{\Omega\left(\mathcal{Q}^{!}\right)}\right)$. The homology of that differential is manifestly the operad $\mathcal{P} \nabla_{0} \mathcal{Q}$, and there is no room for further differentials.

\section{Main result}

Proof of Theorem 1 Let us first establish that $\operatorname{dim}\left(\operatorname{Com} \nabla_{0} \operatorname{Lie}\right)(n)=\operatorname{dim} \operatorname{PreLie}(n)$ for all $n \geq 1$, or in other words

$$
f_{C o m \nabla_{0} L i e}(t)=\sum_{n \geq 1} \frac{\operatorname{dim}\left(\operatorname{Com} \nabla_{0} \operatorname{Lie}\right)(n)}{n !} t^{n}=\sum_{n \geq 1} \frac{\operatorname{dim} \operatorname{PreLie}(n)}{n !} t^{n}=f_{\text {PreLie }}(t) .
$$

It is well known that the right hand side is a solution to the functional equation

$$
f_{\text {PreLie }}(t)=t \exp \left(f_{\text {PreLie }}(t)\right),
$$

which follows either from the rooted trees construction of the operad PreLie as in [4], or from the Koszul duality theory. Thus, it is sufficient to establish that the left-hand side is a solution to the same functional equation. By Proposition 2, the minimal model of Com $\nabla_{0} \mathrm{Lie}$ is generated by

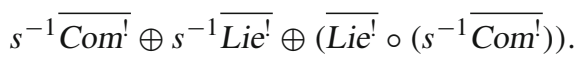

The generating functions of the Euler characteristics of the corresponding operads are, respectively,

$$
\begin{aligned}
& f \overline{\operatorname{Com}^{!}}(t)=-(-\log (1+t)+t)=\log (1+t)-t, \\
& f \frac{\operatorname{Lie} !}{t}(t)=-(\exp (-t)-1+t)=1-t-\exp (-t),
\end{aligned}
$$


so we have

$$
f_{s^{-1}} \overline{\operatorname{Lie}^{!}}(t)=\exp (-t)-1+t, f_{s^{-1}} \overline{C_{o m !} !}(t)=-\log (1+t)+t
$$

and

$$
\begin{aligned}
f \overline{\operatorname{Lie}^{!} \circ\left(s^{-1} \overline{\left.\mathrm{Com}^{!}\right)}(t)\right.} & =1+\log (1+t)-t-\exp (\log (1+t)-t) \\
& =1+\log (1+t)-t-(1+t) \exp (-t),
\end{aligned}
$$

which means that

$$
\begin{aligned}
& f_{s^{-1}} \overline{\operatorname{Com}^{!}}(t)+f_{s^{-1} \overline{\operatorname{Lie}^{!}}}(t)+f \overline{\operatorname{Lie}_{\circ} \circ\left(s^{-1} \overline{C_{0 m} !}\right)}(t) \\
& \quad=-\log (1+t)+t+\exp (-t)-1+t+1+\log (1+t)-t-(1+t) \exp (-t) \\
& \quad=\exp (-t)+t-(1+t) \exp (-t)=t-t \exp (-t) .
\end{aligned}
$$

Finally, it is known [18] that for a minimal model $(\mathcal{T}(\mathcal{E}), d)$ of any operad $\mathcal{P}$, the series $t-f_{\mathcal{E}}(t)$ is the compositional inverse of the series $f_{\mathcal{P}}(t)$, so $t \exp (-t)$ is the compositional inverse of $f_{\mathrm{Com} \nabla_{0} \mathrm{Lie}}(t)$, and

$$
f_{C o m \nabla_{0} L i e}(t) \exp \left(-f_{C o m \nabla_{0} L i e}(t)\right)=t,
$$

which is the same as functional equation (3), as required.

The result we just proved, together with Propositions 1 and 1 , means that we have a diagram of finite-dimensional vector spaces

$$
\operatorname{PreLie}(n) \cong\left(\operatorname{Com} \nabla_{0} \operatorname{Lie}\right)(n) \rightarrow \operatorname{FMan}(n) \rightarrow \operatorname{gr}_{F} \operatorname{PreLie}(n),
$$

where the first and the last term are of the same dimension and all maps are isomorphisms and surjections; hence, all maps must be isomorphisms. In particular, the maps FMan $(n) \rightarrow$ $\operatorname{gr}_{F} \operatorname{PreLie}(n)$ arise from a map of operads, so they assemble into an operad isomorphism. The claims about the dimension and the $S_{n}$-action follow since they are known to hold for the operad PreLie [4], and our filtration is equivariant.

\section{Minimal model of the operad FMan and new constructions of F-manifold algebras}

Recall that our proof of the main result implies that the operad FMan is obtained from operads $\mathcal{P}$ and $\mathcal{Q}$ by an almost distributive law. Using a perturbation argument similar to that in [5], one can establish the following result.

Proposition 3 Suppose that $\mathcal{O}$ is an operad obtained from Koszul operads $\mathcal{P}$ and $\mathcal{Q}$ by an almost distributive law, and suppose further that $\mathcal{O}$ has finite-dimensional components. The shuffle operad $\mathcal{O}$ admits a minimal resolution with generators

$$
s^{-1} \overline{\mathcal{Q}^{!}} \oplus s^{-1} \overline{\mathcal{P} !} \oplus\left(\overline{\mathcal{Q}^{!}} \circ\left(s^{-1} \overline{\mathcal{P} !}\right)\right) .
$$

Let us give a sketch of a proof. The only serious change in comparison with [5] is replacing Gröbner bases with rewriting systems, similar to how methods of Kobayashi [10] extend those of Anick [1].

The first step, analogous to [5, Th. 2.2], which constructs a resolution for any shuffle operad with monomial relations, goes through unchanged. The second step, analogous to [5, 
Th. 4.1], amounts to a perturbation argument which allows one to incorporate lower terms of relations; this argument goes through for rewriting systems without any problem, since in fact it only requires knowing the leading term of each relations for computations and the well-order property for termination of those computations. Since $\mathcal{O}$ is obtained from $\mathcal{P}$ and $\mathcal{Q}$ by an almost distributive law, the proof of Proposition 1 (under our assumption on finite dimensionality of components of the operad $\mathcal{O}$ ) shows that the operads $\mathcal{P} \nabla_{0} \mathcal{Q}$ and $\mathcal{O}$ may be presented by rewriting systems with the same sets of left-hand sides and hence the same associated monomial shuffle operad.

By examining the perturbation argument in [5, Th. 4.1] together with the proof of Proposition 1 , one sees that the differentials induced on the spaces of indecomposable elements of thus obtained resolutions of the operads $\mathcal{P} \nabla_{0} \mathcal{Q}$ and $\mathcal{O}$ have the same homology. Transferring the homotopy cooperad structure from the space of indecomposable elements to homology [6], and recalling Proposition 2, one obtains the claimed result.

Thus, the minimal model of the operad FMan has generators

$$
\left.s^{-1} \overline{\operatorname{Com}^{!}} \oplus s^{-1} \overline{\operatorname{Lie}^{!}} \oplus \overline{\left(\operatorname{Lie}^{!}\right.} \circ\left(s^{-1} \overline{C^{\prime} m^{!}}\right)\right) .
$$

That result is notable in the context of Merkulov's work [19], where the notion of an $F_{\infty^{-}}$ manifold is suggested. The operad controlling $F_{\infty}$-manifolds in the sense of [19] is not cofibrant, since the Jacobi identity for the Lie bracket is suppose to hold strictly, not just up to homotopy (this is an inevitable consequence of thinking of the operation $[-,-]$ as of the bracket of vector fields even when some structures are relaxed up to homotopy).

Our computation confirms that a cofibrant replacement of FMan given by the minimal model is of the "right shape"; its lowest level with respect to the hierarchy of $L_{\infty}$-operations recovers the definition from [19]. This agreement of two results is particularly remarkable in the view of the fact that, unlike Merkulov, we only consider $F$-manifold algebras and ignore the underlying geometry.

As a final remark, let us explain how to construct new examples of $F$-manifold algebras from algebras over operads that are a priori unrelated to geometry. Recall the following definition $[7,15]$.

Definition 5 The operad of pre-Lie commutative algebras is generated by a symmetric binary operation - - - and a binary operation $-\cdot-$ without any symmetry satisfying the following relations:

$$
\begin{aligned}
\left(a_{1} \circ a_{2}\right) \circ a_{3} & =a_{1} \circ\left(a_{2} \circ a_{3}\right), \\
\left(a_{1} \cdot a_{2}\right) \cdot a_{3}-a_{1} \cdot\left(a_{2} \cdot a_{3}\right) & =\left(a_{1} \cdot a_{3}\right) \cdot a_{2}-a_{1} \cdot\left(a_{3} \cdot a_{2}\right), \\
\left(a_{1} \circ a_{2}\right) \cdot a_{3} & =\left(a_{1} \cdot a_{3}\right) \circ a_{2}+a_{1} \circ\left(a_{2} \cdot a_{3}\right) .
\end{aligned}
$$

It turns out that every pre-Lie commutative algebra has a canonical structure of an $F$ manifold algebra.

Proposition 4 In any pre-Lie commutative algebra, the product $\circ$ and the bracket $\left[a_{1}, a_{2}\right]=$ $a_{1} \cdot a_{2}-a_{2} \cdot a_{1}$ satisfy the $F$-manifold algebra identities. 
Proof Associativity and the Jacobi identity are obvious, so we just need to verify the HertlingManin condition. Note that in a pre-Lie commutative algebra, we have

$$
\begin{aligned}
P_{a_{1}}\left(a_{2}, a_{3}\right)= & {\left[a_{1}, a_{2} \circ a_{3}\right]-\left[a_{1}, a_{2}\right] \circ a_{3}-\left[a_{1}, a_{3}\right] \circ a_{2} } \\
= & a_{1} \cdot\left(a_{2} \circ a_{3}\right)-\left(a_{2} \circ a_{3}\right) \cdot a_{1}-\left(a_{1} \cdot a_{2}-a_{2} \cdot a_{1}\right) \circ a_{3} \\
& -\left(a_{1} \cdot a_{3}-a_{3} \cdot a_{1}\right) \circ a_{2} \\
= & a_{1} \cdot\left(a_{2} \circ a_{3}\right)-\left(a_{1} \cdot a_{2}\right) \circ a_{3}-\left(a_{1} \cdot a_{3}\right) \circ a_{2} .
\end{aligned}
$$

From this, the Hertling-Manin condition is quite easy to see:

$$
\begin{aligned}
P_{a_{1} \circ a_{2}}\left(a_{3}, a_{4}\right)= & \left(a_{1} \circ a_{2}\right) \cdot\left(a_{3} \circ a_{4}\right)-\left(\left(a_{1} \circ a_{2}\right) \cdot a_{3}\right) \circ a_{4}-\left(\left(a_{1} \circ a_{2}\right) \cdot a_{4}\right) \circ a_{3} \\
= & \left(a_{1} \cdot\left(a_{3} \circ a_{4}\right)\right) \circ a_{2}+\left(a_{2} \cdot\left(a_{3} \circ a_{4}\right)\right) \circ a_{1}-\left(\left(a_{1} \cdot a_{3}\right) \circ a_{2}\right. \\
& \left.+a_{1} \circ\left(a_{2} \cdot a_{3}\right)\right) \circ a_{4}-\left(\left(a_{1} \cdot a_{4}\right) \circ a_{2}+a_{1} \circ\left(a_{2} \cdot a_{4}\right)\right) \circ a_{3} \\
= & \left(a_{1} \cdot\left(a_{3} \circ a_{4}\right)-\left(a_{1} \cdot a_{3}\right) \circ a_{4}-\left(a_{1} \cdot a_{4}\right) \circ a_{3}\right) \circ a_{2} \\
& +\left(a_{2} \cdot\left(a_{3} \circ a_{4}\right)-\left(a_{2} \cdot a_{3}\right) \circ a_{4}-\left(a_{2} \cdot a_{4}\right) \circ a_{3}\right) \circ a_{1} \\
= & P_{a_{1}}\left(a_{3}, a_{4}\right) \circ a_{2}+P_{a_{2}}\left(a_{3}, a_{4}\right) \circ a_{1},
\end{aligned}
$$

as required.

We conjecture that this construction embeds the operad FMan into the operad of pre-Lie commutative algebras, which may lead to further ways of studying it.

Let us sketch one way to create a somewhat geometric context for this construction. For that, we shall relax the definition of pre-Lie commutative algebras as follows.

Definition 6 The operad of partially commutative pre-Lie pencils is generated by a symmetric binary operation $-\circ-$ and a binary operation $-\cdot-$ without any symmetry satisfying the following relations:

$$
\begin{aligned}
\left(a_{1} \circ a_{2}\right) \circ a_{3} & =a_{1} \circ\left(a_{2} \circ a_{3}\right), \\
\left(a_{1} \cdot a_{2}\right) \cdot a_{3}-a_{1} \cdot\left(a_{2} \cdot a_{3}\right) & =\left(a_{1} \cdot a_{3}\right) \cdot a_{2}-a_{1} \cdot\left(a_{3} \cdot a_{2}\right), \\
\left(a_{1} \circ a_{2}\right) \cdot a_{3}-\left(a_{1} \cdot a_{3}\right) \circ a_{2}-a_{1} \circ\left(a_{2} \cdot a_{3}\right)= & \left(a_{1} \circ a_{3}\right) \cdot a_{2}-\left(a_{1} \cdot a_{2}\right) \circ a_{3} \\
& -a_{1} \circ\left(a_{3} \cdot a_{2}\right) .
\end{aligned}
$$

The reason for the terminology is very simple: a pre-Lie pencil is a vector space with a symmetric binary operation $-0-$ and a binary operation $-\cdot-$ without any symmetry for which the operation $a_{1}, a_{2} \mapsto a_{1} \circ a_{2}+t a_{1} \cdot a_{2}$ is a pre-Lie product for any value of $t$.

It turns out that for any partially commutative pre-Lie pencil, the product $\circ$ and the bracket $\left[a_{1}, a_{2}\right]=a_{1} \cdot a_{2}-a_{2} \cdot a_{1}$ satisfy the $F$-manifold algebra identities; this can be checked by a computation very similar to the one above. A geometric structure behind this definition is that of a flat $F$-manifold [13]. In a forthcoming paper, we shall discuss the connection between this structure and genus zero cohomological field theories, providing a purely algebraic analogue of the connection between flat $F$-manifolds and Frobenius manifolds.

Acknowledgements I am indebted to Yuri Ivanovich Manin for inspiring conversations about $F$-manifolds. Thanks are also due to Frederic Chapoton, Sergei Merkulov, Sergey Shadrin, and Bruno Vallette for useful discussions.

\section{References}

1. Anick, D.J.: On the homology of associative algebras. Trans. Am. Math. Soc. 296(2), 641-659 (1986) 
2. Bergeron, N., Loday, J.-L.: The symmetric operation in a free pre-Lie algebra is magmatic. Proc. Am. Math. Soc. 139(5), 1585-1597 (2011)

3. Bremner, M., Dotsenko, V.: Algebraic Operads: An Algorithmic Companion. CRC Press, Boca Raton (2016)

4. Chapoton, F., Livernet, M.: Pre-Lie algebras and the rooted trees operad. Int. Math. Res. Not. 8, 395-408 (2001)

5. Dotsenko, V., Khoroshkin, A.: Quillen homology for operads via Gröbner bases. Doc. Math. 18, 707-747 (2013)

6. Drummond-Cole, G., Vallette, B.: The minimal model for the Batalin-Vilkovisky operad. Sel. Math. 19(1), 1-47 (2013)

7. Foissy, L.: The Hopf algebra of Fliess operators and its dual pre-Lie algebra. Commun. Algebra 43(10), $4528-4552(2015)$

8. Hertling, C., Manin, Y.I.: Weak Frobenius manifolds. Int. Math. Res. Not. 6, 277-286 (1999)

9. Knuth, D.E., Bendix, P.B.: Simple word problems in universal algebras. In: Computational Problems in Abstract Algebra (Proceedings Conference, Oxford, 1967), pp. 263-297. Pergamon, Oxford (1970)

10. Kobayashi, Y.: Complete rewriting systems and homology of monoid algebras. J. Pure Appl. Algebra 65(3), 263-275 (1990)

11. Livernet, M., Loday, J.-L.: The Poisson operad as a limit of associative operads. Preprint (March 1998)

12. Loday, J.-L., Vallette, B.: Algebraic Operads. Springer, Heidelberg (2012)

13. Manin, Y.I.: $F$-manifolds with flat structure and Dubrovin's duality. Adv. Math. 198(1), 5-26 (2005)

14. Manin, Y.I.: Grothendieck-Verdier duality patterns in quantum algebra. Izv. RAN Ser. matem. 81(4), $158-166$ (2017)

15. Mansuy, A.: Preordered forests, packed words and contraction algebras. J. Algebra 411, 259-311 (2014)

16. Markl, M.: Distributive laws and Koszulness. Ann. Inst. Fourier (Grenoble) 46(2), 307-323 (1996)

17. Markl, M., Remm, E.: Algebras with one operation including Poisson and other Lie-admissible algebras. J. Algebra 299, 171-189 (2006)

18. Markl, M., Remm, E.: (Non-)Koszulness of operads for $n$-ary algebras, galgalim and other curiosities. J. Homotopy Relat. Struct. 10(4), 939-969 (2015)

19. Merkulov, S.A.: Operads, deformation theory and F-manifolds. Asp. Math. 36, 213-251 (2004)

20. Merkulov, S., Vallette, B.: Deformation theory of representations of prop(erad)s. I. J. Reine Angew. Math. 634, 51-106 (2009) 